\title{
A dimeric structure of PD-L1: functional units or evolutionary relics?
}

\author{
Yong Chen ${ }^{1,2,4^{*}}$, Peipei Liu ${ }^{1,2^{*}}$, Feng Gao ${ }^{3}$, Hao Cheng ${ }^{1,2}$, Jianxun Qi $^{1}$, George F. Gao ${ }^{1,2,4,5}(\bowtie)$ \\ ${ }^{1}$ CAS Key Laboratory of Pathogenic Microbiology and Immunology, Institute of Microbiology, Chinese \\ Academy of Sciences (CASPMI), Beijing 100101, China \\ ${ }^{2}$ College of Life Sciences, Graduate University, Chinese Academy of Sciences, Beijing 100049, China \\ ${ }^{3}$ Institute of Biophysics, Chinese Academy of Sciences, Beijing 100101, China \\ ${ }^{4}$ China-Japan Joint Laboratory of Molecular Immunology and Molecular Microbiology, Institute of \\ Microbiology, Chinese Academy of Sciences, Beijing 100101, China \\ ${ }^{5}$ Beijing Institutes of Life Science, Chinese Academy of Sciences, Beijing 100101, China
}

$\bowtie$ Correspondence: gaof@im.ac.cn

Received December 18, 2009; accepted January 7, 2010

\begin{abstract}
PD-L1 is a member of the B7 protein family, most of whose members so far were identified as dimers in a solution and crystalline state, either complexed or uncomplexed with their ligand(s). The binding of PD-L1 with its receptor PD-1 (CD279) delivers an inhibitory signal regulating the $\mathrm{T}$ cell function. Simultaneously with the Garboczi group, we successfully solved another structure of human PD-L1 (hPD-L1). Our protein crystallized in the space group of $\mathrm{C}_{22} 22_{1}$ with two hPD-L1 molecules per asymmetric unit. After comparison of reported B7 structures, we have found some intrinsic factors involved in the interaction of these two molecules. Based on these results, we tend to believe this uncomplexed hPD-L1 structure demonstrated its potential dimeric state in solution, althougt it could just be an evolutionary relic, too weak to be detected under present technology, or still a functional unit deserved our attentions.
\end{abstract}

KEYWORDS PD-L1, crystal structure, dimer, costimulatory molecules, B7 family

\section{INTRODUCTION}

T cells require a co-stimulatory signal to be fully activated in the two-signal model (Lafferty and Cunningham, 1975). The first signal, which gives specificity to the immune response, is provided by the interaction of the antigenic peptide-MHC complex (pMHC) with the T cell receptor (TCR). Co-signal is defined as the second signal because $T$ cell pathways could provide negative as well as positive second signals (Keir et al., 2008). Co-signaling molecules, which can be divided into costimulators and co-inhibitors, are delivered to $\mathrm{T}$ cells by antigen-presenting cells (APCs) to promote or suppress T cell activation, respectively. CD28 family is a set of receptors on $T$ cells while members of $B 7$ family are their ligands, including B7-1, B7-2, PD-L1 (B7-H1), PD-L2 (B7-DC). The B7 family proteins are mainly expressed on $\mathrm{B} / \mathrm{T}$ cells, monocytes, and dendritic cells (DC) (Greenwald et al., 2005). When binding to CD28, B7 family members are co-stimulators for T-cell responses; but they can also act as co-inhibitors by interacting with cytotoxic T lymphocyte antigen 4 (CTLA4) (Chen, 2004). Pathways of the B7:CD28 have key roles in regulating $T$ cell activation and tolerance, and therefore, are promising therapeutic targets (Greenwald et al., 2005).

Programmed death-1 ligand 1 (PD-L1, also called CD274, $\mathrm{B} 7-\mathrm{H} 1$ ) is a member of the B7 family. It is expressed on both lymphoid and nonlymphoid tissues in an inducible manner (Ishida et al., 1992). The binding of PD-L1 with its receptor PD-1 (CD279) delivers an inhibitory signal that regulates the balance between $\mathrm{T}$ cell activation, tolerance, and immunopathology (Keir et al., 2008). PD-L1 can induce and maintain immune tolerance by adjusting the threshold of activation for

"These authors contributed equally to the work. 
thymocytes T cells (Nishimura et al., 1996; Freeman et al., 2000; Fife et al., 2006). In chronic virus infection (such as LCMV, HIV, HCV and HBV), the PD1/PD-L1 pathway plays a key role in regulating the delicate balance between antimicrobial responses and immune-mediated tissue damage (Trautmann et al., 2006; Fisicaro et al., 2007; Zhang et al., 2007; Urbani et al., 2008). Furthermore, when PD-L1 is blocked with antibodies, the anti-tumor immunity is increased (Iwai et al., 2002). In PD-L1-deficient (PD-L1 ${ }^{-1-}$ ) mice, the Tcell responses are augmented and self-reactive $T$ cells appear (Latchman et al., 2004). Recent studies indicated that PD-L1 can also bind with B7-1 to inhibit T cell responses (Keir et al., 2008).

In addition to the functional studies, great efforts have been made to investigate the structures of B7 family. Both biochemical and structure studies have shown that human B7-1 (hB7-1) is a dimer in the crystalline and solution state (Ikemizu et al., 2000). In contrast, hB7-2 exists as a dimer in CTLA-4/B7-2 complex but a monomer in uncomplexed or free state (Zhang et al., 2003). Recently, the David N. Garboczi laboratory presented the structure of the extracellular domain of hPD-L1 and the hybrid samples of hPD-L1 and murine PD1 (mPD1) (Lin et al., 2008). Lazar-Molnar et al. reported the structure of murine PD-L2/PD1 (mPD-L2/mPD1) complex, which shows that both MPD-L2 and MPD1 behave as monomers in the crystal (Lazar-Molnar et al., 2008). It is important to note that the Garboczi group also solved the structure of free state of hPD-L1, with two molecules per asymmetric unit. Detailed analysis was mainly focused on characterizing the binding of the two complex-forming molecules, however, the structure and information revealed from this free state structure of hPD-L1 still remain to be investigated (Lin et al., 2008).

In this report, we crystallized and successfully solved another structure of hPD-L1 simultaneously with the Garboczi group. In our structure, there are also two hPD-L1 molecules per asymmetric unit but they interact with each other through eight hydrogen-bonds interactions. This binding presents a rotating angle of the two molecules. We also found the specific hinge angle between $\operatorname{lgV} / \operatorname{lgC}$ domains, which is mainly formed by a polar contact in the hinge region. Based on this structure, we hypothesize two possibilities: 1) the dimeric molecules of PD-L1 with "weak" interaction as functional units are formed in nature or 2) this is simply an euolutionary relic of B7 family.

\section{RESULTS}

\section{Overall structure}

The extracellular domain of human PD-L1 (hPD-L1) was crystallized in the space group of $\mathrm{C} 222_{1}$, with unit cell parameters shown in Table 1. In the crystal lattice, two copies of PD-L1 together form each asymmetric unit, termed as molecule $\mathbf{a}$ and $\mathbf{b}$, respectively (Fig. 1A).
Table 1 X-ray diffraction data processing and refinement statistics Data Collecting

space group

$\mathrm{C} 222_{1}$

unit cell dimensions $(a, b, c)$

$71.87,91.37,140.32$

unit cell dimensions $(\alpha, \beta, y)$

$90.00,90.00,90.00$

resolution range $(\AA)$

number of unique reflections

$50.0-2.60(2.70-2.60)^{\mathrm{a}}$

number of molecule in the asymmetric unit

36104

average redundancy

2

completeness (\%)

$5.7(3.6)$

$\mathrm{R}_{\text {merge }}(\%)$

$91.1(98.1)$

I/ $\sigma$

8.3 (29.7)

$13.5(5.2)$

\section{Refinement}

resolution $(\AA)$

$15.0-2.70$

completeness (\%)

97.6

R-factor (\%)

22.6

$\mathrm{R}_{\text {free }}{ }^{\mathrm{b}}(\%)$

29.6

r.m.s.d. from restraint target values: bond lengths $(\AA) \quad 0.015$

bond angles (degrees) $\quad 1.73$

ramachandran plot quality:

residues in most favored regions $\quad 324 \quad 82.9 \%$

residues in additional allowed $\quad 60 \quad 15.3 \%$

residues in generously allowed $\quad 7 \quad 1.8 \%$

residues in disallowed regions

$0 \quad 0$

\footnotetext{
${ }^{a}$ Values in parentheses are given for the highest resolution shell.

${ }^{b} R_{\text {free }}$ is calculated over reflections in a test set (5\%) not included in atomic refinement.
}

PD-L1 contains two anti-parallel $\beta$ sandwich immunoglobulin superfamily (IgSF) domains, D1, D2 from N'- to C'terminus. The domain 1 (D1) of PD-L1 has V-set topology (IgV), with $\beta$ strands forming BED and AGFCC'C" sheets. Short regions of $3_{10}$ helix are found to connect the B-C strands, C"-D strands, D-E strands and E-F strands, respectively. The membrane proximal domain (domain 2, D2) of PD-L1 is formed by ABED and CFG $\beta$-sheets, which is typical of $\mathrm{C} 1$-set domains $(\mathrm{IgC})$. The single domain structure of PD-L1 in this crystal is highly similar to that of the hPD-L1 monomer complexed with murine PD1 (mPD1) (PDB: 3BIK, chain $A$ ), with root mean square deviations (r.m.s.d.) of $0.489 \AA$ for domain 1 (D1) and $0.432 \AA$ for domain 2 (D2), respectively.

Of the known structures of B7 family, B7-1 is a dimer and B7-2 in complex with CTLA4 is also reported as a dimer in the crystals; furthermore, the steric arrangements of the two monomers in the asymmetric unit are also similar. As shown in Fig. 1A, the two molecules in B7-1 structure are parallel to each other. However, in B7-2, there is a little rotation between the two monomers with a torsion angle of only $\sim 10^{\circ}$. Nevertheless, in comparison with B7-1, the two PD-L1 molecules in one asymmetric unit are not located in a horizontal line and molecule a is observed to rotate up to $30^{\circ}$ 


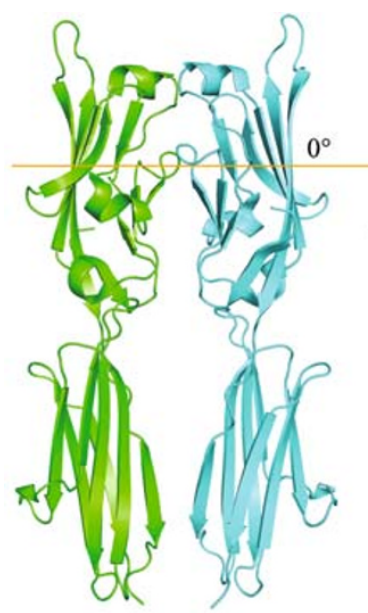

B7-1

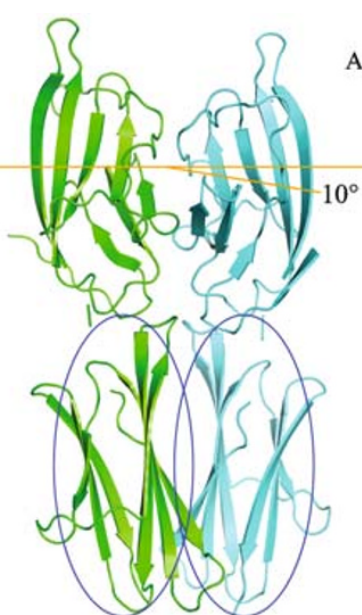

B7-2

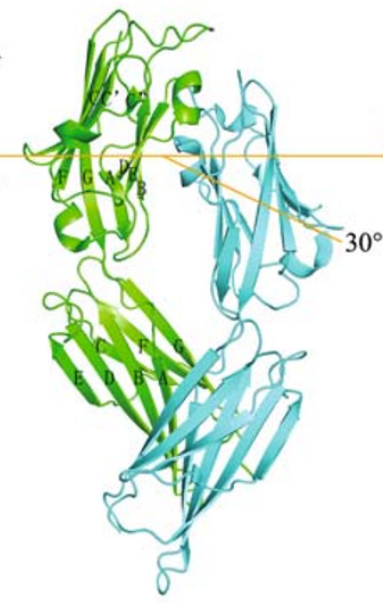

B7-H1/PD-L1

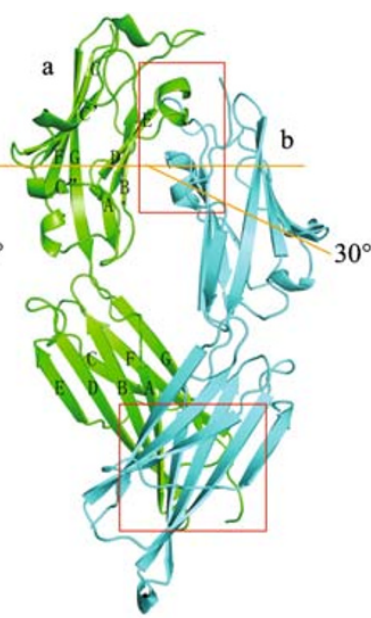

B7-H1/PD-L1

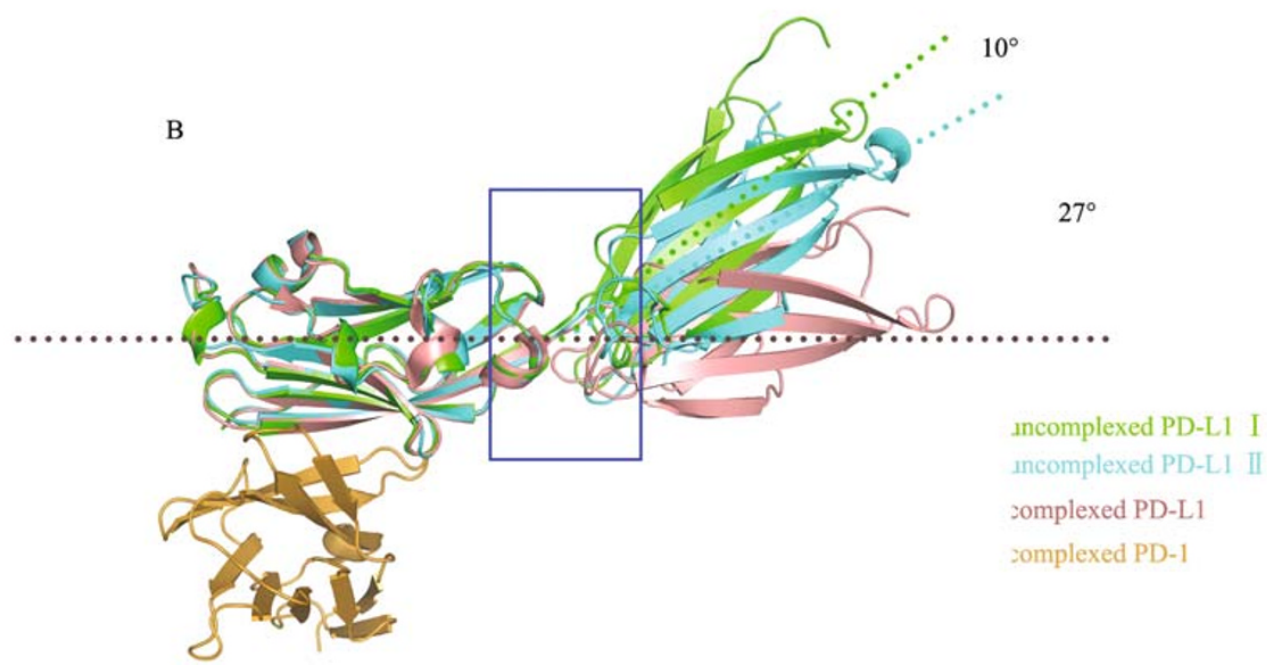

Figure 1. Dimeric structure of human PD-L1 and its comparison with B7 family structures. (A) Presentation of solved B7 family structures: B7-1 (PDB 1/8L); B7-2 (PDB 1/85); PD-L1 from the Garboci group (PDB 3BIS); PD-L1 solved by us (molecule a and $\mathbf{b}, \mathrm{PDB} 3 \mathrm{FN} 3$ ). As the structure is still unknown, the domain 2 of B7-2 was modeled based on B7-1 corresponding domain and circled blue. The areas boxed in red were zoomed in for details in Fig. 3. (B) Superposition of uncomplexed and complexed hPD-L1 with domain 1 fixed. The area boxed in blue was zoomed in for details in Fig. 2.

relative to molecule b (Fig. 1A). Along with the hPD-L1/mPD1 complex structure, The. Garboczi group also revealed a freestate structure of hPD-L1 (PDB: 3BIS) with the same angle as observed in our structure. However, using either the 3BIS or single chain as search probe, the molecular replacements during structure determination are both unsuccessful as indicated in Materials and Methods. This would suggest that the local structure differs between our coordinate and 3BIS, which is consistent with the r.m.s.d. of $0.512 \AA$ for these two structures.

\section{Turning angle between $\lg V$ and $\lg C$ domains of PD-L1}

As indicated by the structures of hPD-L1 and mPD-L2 complexed with mPD1 (PDB: 3BIK, Lin et al., 2008; PDB:
3BP5, Lazar-Molnar et al., 2008), IgV (D1) and IgC (D2) are arranged almost in a straight line. However, the uncomplexed hPD-L1 and the complexed hPD-L1 differ in the hinge angle between $\lg V$ and $\lg C$ by $\sim 30^{\circ}$. Nevertheless, the hinge angle of hPD-L1 molecule $\mathbf{a}$ is larger than molecule b by $10^{\circ}$ (Fig. 1B).

Previous studies have shown that the interactions between neighboring Ig domains mainly involve hydrophobic residues; therefore, the angle between the Ig domains is relatively fixed by the locations and contacts of these hydrophobic residues (Chen et al., 2009). The PD-L1's hinge angle of IgV/lgC domains indicates that the interaction between these two domains is weak. This is also confirmed by the solvent accessible buried area of the $\operatorname{lgV} / \operatorname{lgC}$ domains, $814.8 \AA^{2}$, which is much lower than reported values of some other $2-\mathrm{lg}$ structures, such as ILT2 (957 $\AA^{2}$ ) (Willcox et al., 2002), 
KIR2DL2 (949 $\left.\AA^{2}\right)$ (Snyder et al., 1999), NKp46 (1021 $\left.\AA^{2}\right)$ (Foster et al., 2003).

On the other hand, detailed analysis of the interface between such $\lg V$ and $\lg C$ domains demonstrated that the angle of these two domains was not randomly formed. The binding of Glu31 in IgV to Asn 135 of $\lg C$ domain with a strong polar contact as this hinge area represents one of the most significant differences between dimeric (uncomplexed) PD-L1 and complexed PD-L1 (Fig. 2). This bond dragged the IgC domain off the axle wire of the IgV domain, which may be a reason for the turning pattern in the dimeric PD-L1.

\section{Dimeric interface of PD-L1 in the crystal}

For $B 7-1$, the dimer is formed mainly by the interaction between two D1 domains, which buries $1220 \AA^{2}$ of surface area for each molecule. The D1 interaction is mediated by a relatively flat surface formed by the $B, C$ ", $D$, and $E \beta$-strands with hydrophobic residues (Val11, Val22, Gly45, Met47, Ile58, Asp60, lle61, Thr62 and Leu70). There is no contact between the $\mathrm{D} 2$ domains of each molecule.

Compared with B7-1, B7-2 dimer interface is predominantly formed by the B, E and D strands and the BC, C'D and DE loops from the back sheet of each monomer, and buries a total accessible surface area of $1405 \AA^{2}$. When focusing on the residues forming the dimer interface, different from B7-1, most residues of the B7-2 dimer interface are hydrophilic.

In contrast with the B7-1 and B7-2's dimerization that is mainly mediated through D1/D1 interface, the two molecules in our structure interact with each other through two independent intermolecular interfaces. The total buried accessible surface area is $859.1 \AA^{2}$ (interface 1, D1/D1, Fig. 3A) and $1292.2 \AA^{2}$ (interface 2, D2/D2, Fig. 3B), respectively. This is consistent with the existence of more contacting residues in the D2/D2 interface as shown in Fig. 3. This interface area $\left(2151.3 \AA^{2}\right)$ is much larger than that of homodimer of B7-1 or B7-2 (Schwartz et al., 2001; Stamper et al., 2001). Furthermore, similar to the B7-2 dimer interface, residues of the PD-L1/PD-L1 interface are hydrophilic (Fig. 3).

As we discussed above, the turning angle of $\lg V$ and $\lg C$ was determined by the polar contacts of Glu31 and Asn135. Furthermore, this angle can introduce a favorite position for the PD-L1 dimeric docking, as we see in this structure.

\section{EGS cross-linking}

In order to examine whether there is any dimer of PD-L1 existing in vitro, an EGS cross-linking experiment was performed as previously described (Chen et al., 2007). As the concentration of EGS increases, the protein band corresponding to the dimer form of PD-L1 became stronger (Fig. 4). It demonstrates that hPD-L1 has a tendency toward dimer formation in solution.

\section{DISCUSSION}

\section{Functional units or evolutionary relics?}

Previous studies of B7 family members indicated that B7-1 and $B 7-2$ behave as dimer when interacting with their ligands CTLA-4 (Schwartz et al., 2001; Stamper et al., 2001). Nevertheless, without ligands, B7-1 dimer was also reported both in solution and in the crystal state (Ikemizu et al., 2000). On the other hand, the monomeric form of B7-2 has been observed both in crystal (Zhang et al., 2003) and in solution,

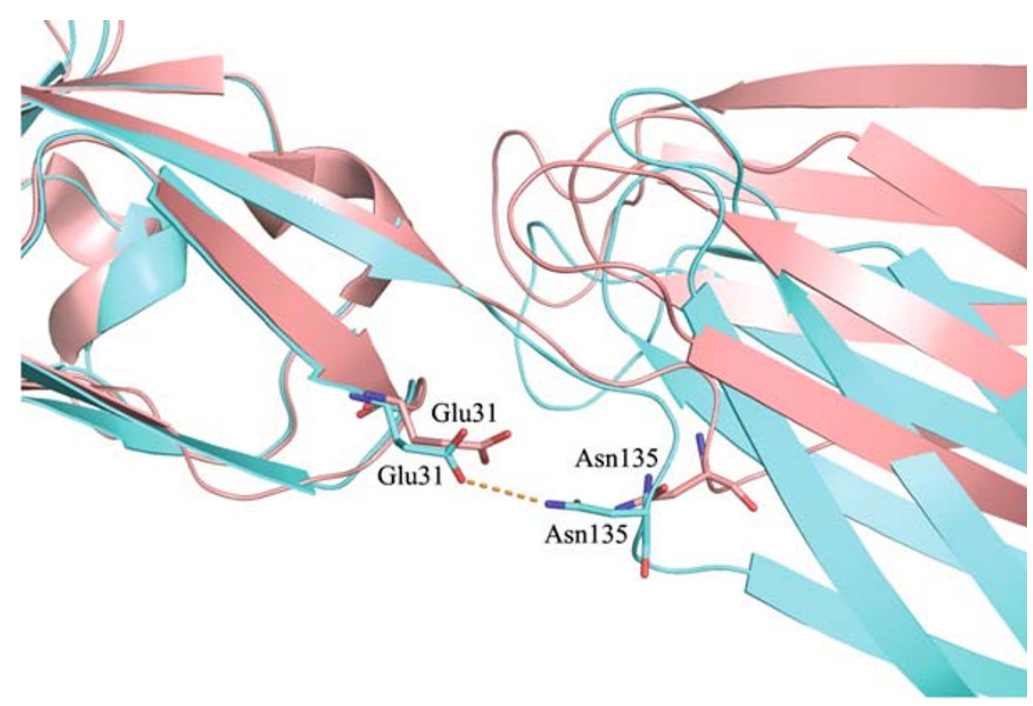

Figure 2. Comparison of the hinge region and the turning angle of uncomplexed and complexed hPD-L1. The polar contact between the $\operatorname{lgV}$ domain and $\operatorname{lgC}$ domain of complexed (magenta) and uncomplexed (cyan) was shown as broken orange lines. 

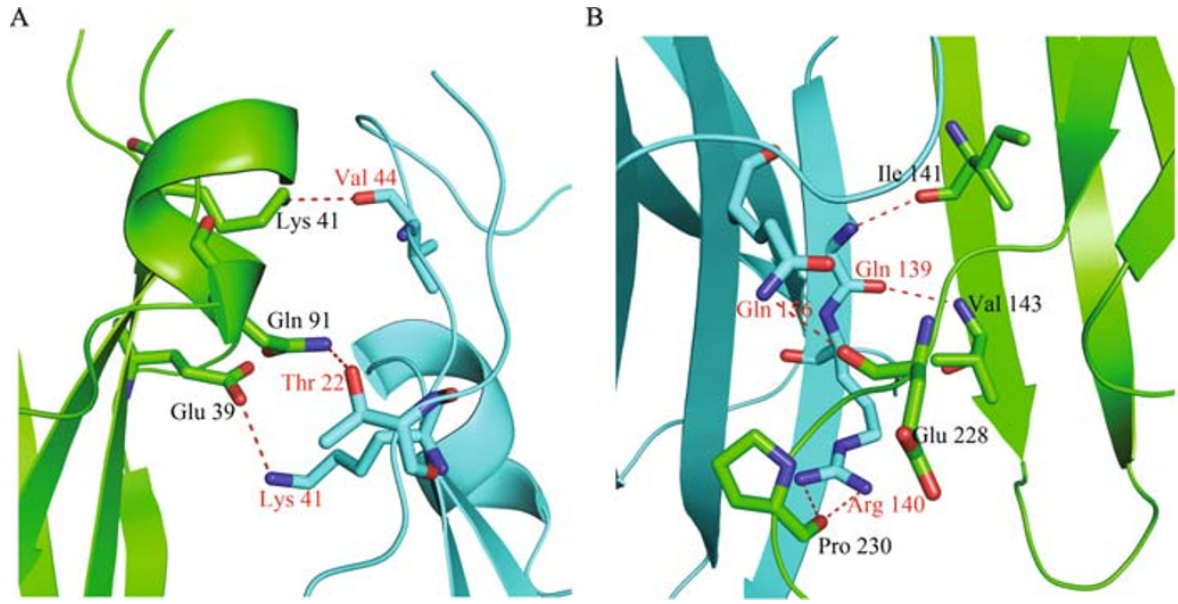

Figure 3. Zoom-in for the details of the PD-L1 D1/D1 (IgV) and D2/D2 (IgC) interface, respectively. Hydrogen bonds involved in the interface were shown as broken red lines. (A) Zoom-in image for the detailed view of the D1/D1 interface shows that there are three hydrogen bonds for D1/D1 binding. (B) Detailed view of the D2/D2 interface shows that five hydrogen bonds connect the two D2 domains.

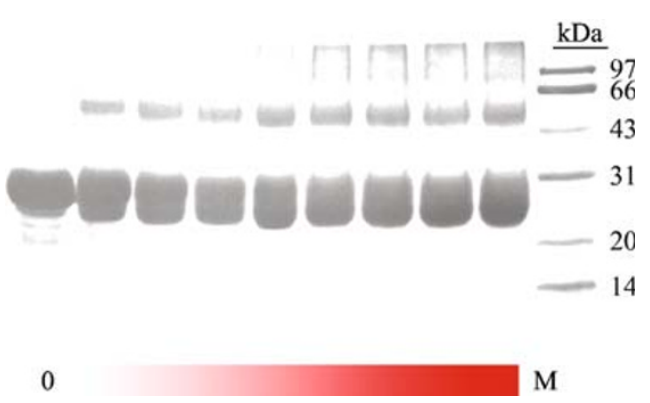

Figure 4. EGS cross linking of hPD-L1. The number 0 and the color density of the red line indicate the final concentration gradient of ethyleneglycol bis (-succinimidylsuccinate) (EGS). Lane M contains protein marker with 97, 66, 43, 31, 20 and 14 $\mathrm{kDa}$ bands from top to bottom.

by gel filtration chromatography and sedimentation equilibrium experiments. This monomeric form of B7-2 can also bind tightly to bacterially expressed monomeric and human disulfide-linked homodimeric CTLA-4 (Zhang et al., 2002).

Here, we presented another hPD-L1 structure. In contrast to hPD-L1 complexed with its ligand PD1, free state hPD-L1 in crystal behaves as two contacting molecules like a dimer with a rotating angle. B7-2 dimer behaves with the similar rotation, even though the angle is relatively smaller $\left(10^{\circ}\right.$, Fig. 1A). Point-worthily, not only the contacting residues, but also the dimeric interface of hPD-L1 displays a hydrophilic and electron complementarity dimer interface (Fig. 5A), just like the B7-2 dimer observed in the B7-2/CTLA-4 complex.

It is reported that the dimerization of $B 7$ isoforms may be favored at the T cell/APC interface, because this twodimensional arrangement of B7 molecules facilitate the oligomerization, compared with the random distribution of individual B7 molecules in solution (Zhang et al., 2003). Furthermore, the ligands of B7 proteins, CD28, CTLA-4 and/ or PD1, which are enriched in the immunological synapse (Bromley et al., 2001; Egen and Allison, 2002), may bind and recruit $B 7$ molecules, bringing them into proximity, increasing their local concentrations, and further constraining them in a more productive orientation for dimerization (Zhang et al., 2003). These dimeric molecules continue to recruit their ligands, and consequently, generate a tandem repeat in the cell-cell interface. Based on the data of hPD-L1/mPD1 and $\mathrm{mPD}-\mathrm{L} 2 / \mathrm{mPD} 1$, we also proposed a model that PD1 binds to the dimeric $\mathrm{PD}-\mathrm{L} 1$, which is reasonable as no steric hindrance exit (Fig. 5B).

Taken together, even though some biochemical and crystallographic analyses observed the monomeric form of hPD-L1 (Lin et al., 2008; Simon Davis of Oxford, personal communication), we cannot yet rule out the existence of hPDL1 dimer, which may be either a functional unit in immunological synapse formation or a revolution relics of B7 family. To address the first possibility, the future new technology might help detect this dimerization in vitro.

\section{MATERIALS AND METHODS}

\section{Cloning, expression and purification of PD-L1}

Nucleotides covering amino acids Phe19 to Arg238 of the ectodomain of hPD-L1 was cloned from human lymphocyte cDNA library mRNA (Stratagene) with the following primers:

PD-L1-F, 5'-GGAATTCCATATGTTTACTGTCACGGTTCCCAAGG-3';

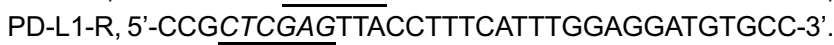

Inclusion bodies of hPD-L1 were prepared, and its protein was renatured and purified as previously described (Gao et al., 1997, 1998; Chen et al., 2007). 

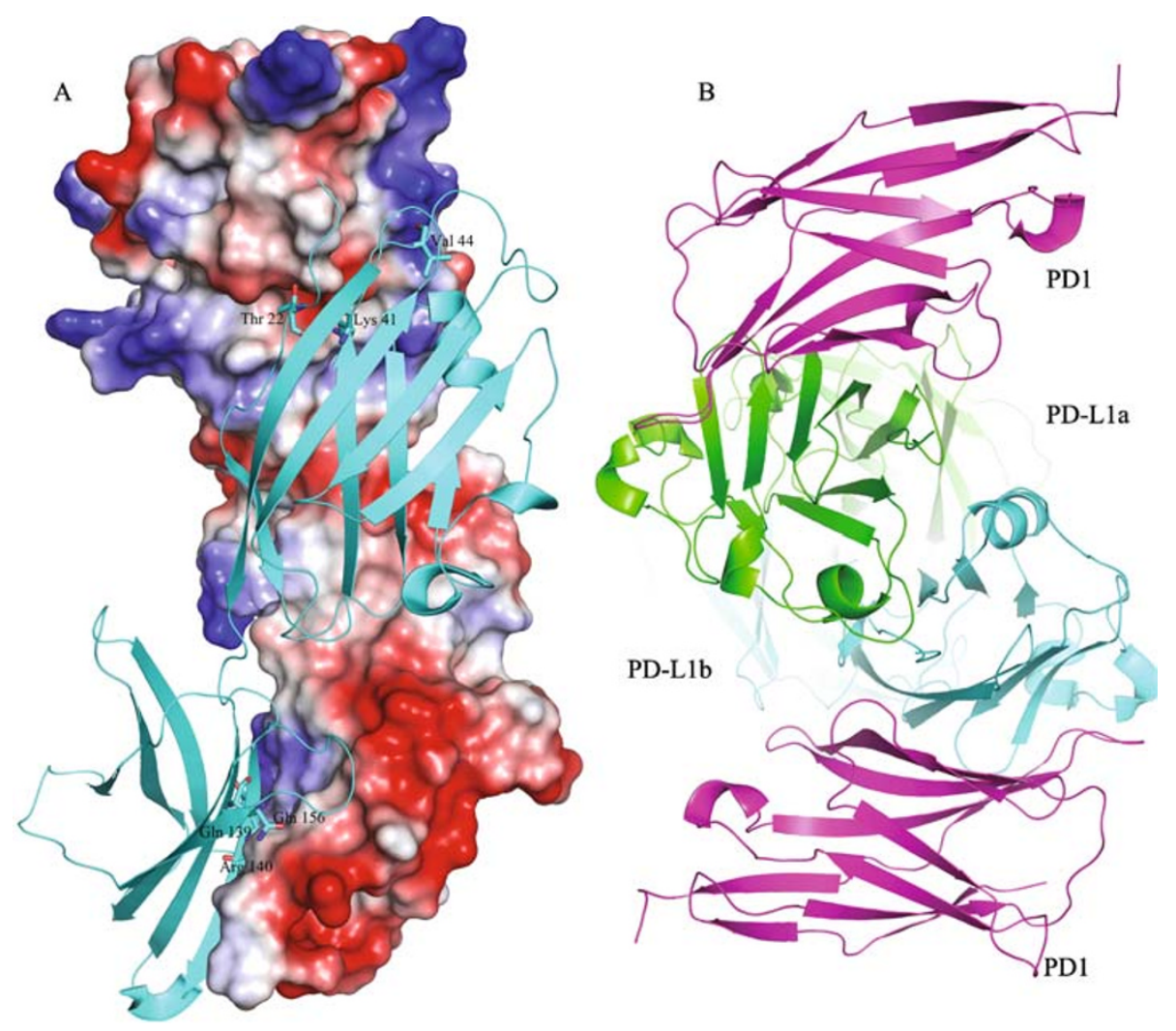

Figure 5. Overview of the highly charged dimeric interface of hPD-L1 and the modeling of hPD-L1/hPD1 complex. (A) The binding mode of hPD-L1 with molecule $\mathbf{b}$ shown in ribbon and the surface of molecule a colored by electrostatic potential (positive, blue; negative, red). The contacting residues were shown in sticks. (B) Modeling for the potential dimeric binding pattern of hPD-L1/ hPD1 complex based on the hPD-L1/mPD1 structure (PDB 3BIK) and the structure of hPD-L1 (PDB 3NF3).

\section{Crystallization of PD-L1, data collection and processing}

Ideal crystals of hPD-L1 were grown from a 1:1 mixture of the protein solution $(20 \mathrm{mg} / \mathrm{mL})$ with crystallization reagent of $15 \%$ PEG3350 (w/ v), $0.2 \mathrm{M}$ ammonium actate $\left(\mathrm{NH}_{4} \mathrm{Ac}\right)$, and $\left.0.1 \mathrm{M} \mathrm{BIS-Tris} \mathrm{(} \mathrm{pH} 5.5\right)$ at $18^{\circ} \mathrm{C}$ by the hanging drop vapor diffusion method. Data were collected using a Rigaku MicroMax007 rotating-anode X-ray generator ( $\mathrm{Cu} K \alpha, \lambda=1.5418 \AA$ ) equipped with an R-AXIS VII ++ imageplate detector. Data were processed and scaled using HKL2000 (Otwinowski and Minor, 1997).

\section{Structure determination of PD-L1}

Data were analyzed by molecular replacement using Phaser (McCoy, 2007) in the CCP4 package (CCP4, 1994), taking separated domains as probe derived from hPD-L1 structure from David N. Garboczi's group (PDB code: 3BIS) (Lin et al., 2008). Final rounds of refinement resulted in a final Rcryst of $22.6 \%\left(R_{\text {free }}=29.6 \%\right)$ for all data between $20.0 \AA$ and $2.70 \AA$.

Buried surface areas were calculated using SURFACE (CCP4, $1994)$ with a $1.4 \AA$ probe radius. The PyMOL Molecular Graphics System (DeLano Scientific, http://www.pymol.org) was used to prepare figures. Geometry of the refined structure was validated according to
Ramachandran plot criteria (Lovell et al., 2003). The data collection and refinement statistics of the structure are shown in Table 1.

\section{EGS cross-linking}

The purified hPD-L1 proteins were cross-linked with ethyleneglycol bis(-succinimidylsuccinate) (EGS) (Sigma) as previously reported (Zhu et al., 2005; Chen et al., 2007). Cross-linked products were analyzed by reducing SDS-PAGE ( $12 \%$ polyacrylamide gel).

\section{DATA DEPOSITION}

Atomic coordinates have been deposited in the Protein Data Bank (PDB http://www.rcsb.org/pdb ) under accession code 3FN3.

\section{ACKNOWLEDGEMENTS}

We thank Mr. Christopher Pannell for his critical reading of the manuscript and Dr. Fuliang Chu for his valuable suggestions.

This work was supported by a grant from Ministry of Science and Technology (MOST) of China for the basic research program 973, Grant No. 2006CB504204; a grant from National Natural Science Foundation (NSFC) of China, Grant No. 30671903 and a grant from Chinese Academy of Sciences (CAS) Knowledge Innovation Project, 
Grant No. KSCX2-SW-227. G.F.G. is a distinguished young investigator of the NSFC (Grant No. 30525010). Y.C. is a Ph.D. student supported by Science Innovation Project, Graduate University of Chinese Academy of Sciences (GUCAS), No. 0729031EE1. The China-Japan Joint Laboratory of Molecular Immunology and Molecular Microbiology is partlially supported by Japan MEXT (Ministry of Education, Culture, Sports, Science and Technology).

\section{ABBREVIATIONS}

APC, antigen-presenting cell; CTLA4, cytotoxic T lymphocyte antigen 4; HBV, hepatitis B virus; HCV, hepatitis C virus; HIV, human immunodeficiency virus; LCMV, lymphocytic choriomeningitis virus; PD-1, Programmed Death-1; PDL1, PD-1 ligand 1; pMHC, antigenic peptide major histocompatibility complex; r.m.s.d., root mean square deviations; TCR, T cell receptor

\section{REFERENCES}

Bromley, S.K., laboni, A., Davis, S.J., Whitty, A., Green, J.M., Shaw, A.S., Weiss, A., and Dustin, M.L. (2001). The immunological synapse and CD28-CD80 interactions. Nat Immunol 2, 1159 1166.

CCP4 (1994). The CCP4 suite: programs for protein crystallography. Acta Crystallogr D Biol Crystallogr 50, 760-763.

Chen, L.P. (2004). Co-inhibitory molecules of the B7-CD28 family in the control of T-cell immunity. Nat Rev Immunol 4, 336-347.

Chen, Y., Chu, F., Gao, F., Zhou, B., and Gao, G.F. (2007). Stability engineering, biophysical, and biological characterization of the myeloid activating receptor immunoglobulin-like transcript 1 (ILT1/ LIR-7/LILRA2). Protein Expr Purif 56, 253-260.

Chen, Y., Gao, F., Chu, F., Peng, H., Zong, L., Liu, Y., Tien, P., and Gao, G.F. (2009). Crystal structure of myeloid cell activating receptor leukocyte Ig-like receptor A2 (LILRA2/ILT1/LIR-7) domain swapped dimer: molecular basis for its non-binding to $\mathrm{MHC}$ complexes. J Mol Biol 386, 841-853.

Egen, J.G., and Allison, J.P. (2002). Cytotoxic T lymphocyte antigen-4 accumulation in the immunological synapse is regulated by TCR signal strength. Immunity 16, 23-35.

Fife, B.T., Guleria, I., Gubbels Bupp, M., Eagar, T.N., Tang, Q., BourJordan, H., Yagita, H., Azuma, M., Sayegh, M.H., and Bluestone, J. A. (2006). Insulin-induced remission in new-onset NOD mice is maintained by the PD-1-PD-L1 pathway. J Exp Med 203, 2737-2747.

Fisicaro, P., Boni, C., Valdatta, C., Bertoletti, A., Schivazappa, S., Giuberti, T., Cavalli, A., Missale, G., and Ferrari, C. (2007). PD-1/ PD-L1 blockade can improve the HBV-specific T cell function in chronic hbv infection. J Hepatol 46, S56-S56.

Foster, C.E., Colonna, M., and Sun, P.D. (2003). Crystal structure of the human natural killer (NK) cell activating receptor NKp46 reveals structural relationship to other leukocyte receptor complex immunoreceptors. J Biol Chem 278, 46081-46086.

Freeman, G.J., Long, A.J., Iwai, Y., Bourque, K., Chernova, T., Nishimura, H., Fitz, L.J., Malenkovich, N., Okazaki, T., Byrne, M.C., et al. (2000). Engagement of the PD-1 immunoinhibitory receptor by a novel B7 family member leads to negative regulation of lymphocyte activation. J Exp Med 192, 1027-1034.

Gao, G.F., Gerth, U.C., Wyer, J.R., Willcox, B.E., O'Callaghan, C.A.,
Zhang, Z., Jones, E.Y., Bell, J.I., and Jakobsen, B.K. (1998). Assembly and crystallization of the complex between the human $T$ cell coreceptor CD8alpha homodimer and HLA-A2. Protein Sci 7 , 1245-1249.

Gao, G.F., Tormo, J., Gerth, U.C., Wyer, J.R., McMichael, A.J., Stuart, D.I., Bell, J.I., Jones, E.Y., and Jakobsen, B.K. (1997). Crystal structure of the complex between human CD8 alpha and HLA-A2. Nature 387, 630-634.

Greenwald, R.J., Freeman, G.J., and Sharpe, A.H. (2005). The B7 family revisited. Annu Rev Immunol 23, 515-548.

Ikemizu, S., Gilbert, R.J., Fennelly, J.A., Collins, A.V., Harlos, K., Jones, E.Y., Stuart, D.I., and Davis, S.J. (2000). Structure and dimerization of a soluble form of B7-1. Immunity 12, 51-60.

Ishida, Y., Agata, Y., Shibahara, K., and Honjo, T. (1992). Induced expression of $\mathrm{Pd}-1$, a novel member of the immunoglobulin gene superfamily, upon programmed cell-death. EMBO J 11, 3887-3895.

Iwai, Y., Ishida, M., Tanaka, Y., Okazaki, T., Honjo, T., and Minato, N. (2002). Involvement of PD-L1 on tumor cells in the escape from host immune system and tumor immunotherapy by PD-L1 blockade. Proc Natl Acad Sci U S A 99, 12293-12297.

Keir, M.E., Butte, M.J., Freeman, G.J., and Sharpel, A.H. (2008). PD1 and its ligands in tolerance and immunity. Annu Rev Immunol 26, 677-704.

Lafferty, K.J., and Cunningham, A.J. (1975). A new analysis of allogeneic interactions. Aust J Exp Biol Med 53, $27-42$.

Latchman, Y.E., Liang, S.C., Wu, Y., Chernova, T., Sobel, R.A., Klemm, M., Kuchroo, V.K., Freeman, G.J., and Sharpe, A.H. (2004). PD-L1-deficient mice show that PD-L1 on T cells, antigenpresenting cells, and host tissues negatively regulates $\mathrm{T}$ cells. Proc Natl Acad Sci U S A 101, 10691-10696.

Lazar-Molnar, E., Yan, Q., Cao, E., Ramagopal, U., Nathenson, S.G., and Almo, S.C. (2008). Crystal structure of the complex between programmed death-1 (PD-1) and its ligand PD-L2. Proc Natl Acad Sci U S A 105, 10483-10488.

Lin, D.Y., Tanaka, Y., Iwasaki, M., Gittis, A.G., Su, H.P., Mikami, B., Okazaki, T., Honjo, T., Minato, N., and Garboczi, D.N. (2008). The PD-1/PD-L1 complex resembles the antigen-binding Fv domains of antibodies and T cell receptors. Proc Natl Acad Sci U S A 105, 3011-3016.

Lovell, S.C., Davis, I.W., Arendall, W.B., 3rd, de Bakker, P.I., Word, J. M., Prisant, M.G., Richardson, J.S., and Richardson, D.C. (2003). Structure validation by Calpha geometry: phi,psi and Cbeta deviation. Proteins 50, 437-450.

McCoy, A.J. (2007). Solving structures of protein complexes by molecular replacement with Phaser. Acta Crystallogr D Biol Crystallogr 63, 32-41.

Nishimura, H., Agata, Y., Kawasaki, A., Sato, M., Imamura, S., Minato, N., Yagita, H., Nakano, T., and Honjo, T. (1996). Developmentally regulated expression of the $\mathrm{PD}-1$ protein on the surface of double-negative(CD4-CD8-) thymocytes. Int Immunol 8, 773-780.

Otwinowski, Z., and Minor, W. (1997). Processing of X-ray diffraction data collected in oscillation mode. Methods Enzymol 276, 307-326.

Schwartz, J.C., Zhang, X., Fedorov, A.A., Nathenson, S.G., and Almo, S.C. (2001). Structural basis for co-stimulation by the human CTLA-4/B7-2 complex. Nature 410, 604-608. 
Snyder, G.A., Brooks, A.G., and Sun, P.D. (1999). Crystal structure of the HLA-Cw3 allotype-specific killer cell inhibitory receptor KIR2DL2. Proc Natl Acad Sci U S A 96, 3864-3869.

Stamper, C.C., Zhang, Y., Tobin, J.F., Erbe, D.V., Ikemizu, S., Davis, S. J., Stahl, M.L., Seehra, J., Somers, W.S., and Mosyak, L. (2001). Crystal structure of the B7-1/CTLA-4 complex that inhibits human immune responses. Nature 410, 608-611.

Trautmann, L., Janbazian, L., Chomont, N., Said, E.A., Gimmig, S., Bessette, B., Boulassel, M.R., Delwart, E., Sepulveda, H., Balderas, R.S., et al. (2006). Upregulation of PD-1 expression on HIV-specific CD8(+) T cells leads to reversible immune dysfunction. Nat Med 12, 1198-1202.

Urbani, S., Amadei, B., Tola, D., Pedrazzi, G., Sacchelli, L., Cavallo, M.C., Orlandini, A., Missale, G., and Ferrari, C. (2008). Restoration of HCV-specific T cell functions by PD-1/PD-L1 blockade in HCV infection: Effect of viremia levels and antiviral treatment. J Hepatol 48, 548-558.

Willcox, B.E., Thomas, L.M., Chapman, T.L., Heikema, A.P., West, A. P., Jr., and Bjorkman, P.J. (2002). Crystal structure of LIR-2 (ILT4) at 1.8 A: differences from LIR-1 (ILT2) in regions implicated in the binding of the human cytomegalovirus class I MHC homolog UL18. BMC Struct Biol 2, 6-15.

Zhang, J.Y., Zhang, Z., Wang, X., Fu, J.L., Yao, J., Jiao, Y., Chen, L., Zhang, H., Wei, J., Jin, L., et al. (2007). PD-1 up-regulation is correlated with HIV-specific memory CD8 + T-cell exhaustion in typical progressors but not in long-term nonprogressors. Blood 109, 4671-4678.

Zhang, X., Schwartz, J.C., Almo, S.C., and Nathenson, S.G. (2002). Expression, refolding, purification, molecular characterization, crystallization, and preliminary X-ray analysis of the receptor binding domain of human B7-2. Protein Expr Purif 25, 105-113.

Zhang, X., Schwartz, J.C., Almo, S.C., and Nathenson, S.G. (2003). Crystal structure of the receptor-binding domain of human B7-2: insights into organization and signaling. Proc Natl Acad Sci U S A 100, 2586-2591.

Zhu, J., Jiang, X., Liu, Y., Tien, P., and Gao, G.F. (2005). Design and characterization of viral polypeptide inhibitors targeting Newcastle disease virus fusion. J Mol Biol 354, 601-613. 